\title{
Interferometric method of determining the refraction index of two-dimensional sonic crystals
}

\author{
V. Romero-García \\ Grupo de Acústica Arquitectónica y del Medio Ambiente, Departamento de Física Aplicada, Universidad Politécnica de Valencia, Cno. \\ de Vera s/n, 46022 Valencia, Spain \\ E. Fuster-Garcia \\ Grupo de Acústica Arquitectónica y del Medio Ambiente, Departamento de Física Aplicada, Universidad Politécnica de Valencia, Cno. \\ de Vera s/n, 46022 Valencia, Spain \\ L. M. García-Raffi \\ Instituto de Matemática Pura y Aplicada, Departamento de Matemática Aplicada, Universidad Politécnica de Valencia, Cno. de Vera s/n, \\ 46022 Valencia, Spain \\ J. V. Sánchez-Pérez \\ Grupo de Acústica Arquitectónica y del Medio Ambiente, Departamento de Física Aplicada, Universidad Politécnica de Valencia, Cno. \\ de Vera s/n, 46022 Valencia, Spain \\ A. Uris \\ Grupo de Acústica Arquitectónica y del Medio Ambiente, Departamento de Física Aplicada, Universidad Politécnica de Valencia, Cno. \\ de Vera s/n, 46022 Valencia, Spain
}

(Received 1 June 2006; revised manuscript received 9 November 2006; published 15 June 2007)

\begin{abstract}
Sonic crystals are defined as materials consisting of periodic distributions of acoustic scatterers in another medium with different elastic properties. These materials present two main acoustical features: they present ranges of frequencies where the acoustic wave propagation is forbidden and, moreover, they can be used to construct acoustical refractive devices. We present here an interferometric method of characterizing directly the refraction index of these materials. We focused our research on sonic crystals constructed with a periodic distribution of rigid cylinders in air. The interferometric method used is based on the acoustical analog of an optical device called Fresnel's biprism. Our numerical predictions are in good agreement with the experimental results obtained and allow us to estimate the refraction index for very low frequencies. Moreover, in this range we show the nonlinear behavior in the dispersion relation refraction index versus frequency. Finally, we analyze the relationship between the refraction index and some typical sonic crystal parameters. In view of the results we can conclude that this method offers certain advantages with respect to the classical method used to obtain the refraction index in sonic crystals with airborne propagation: the phase delay method.
\end{abstract}

DOI: 10.1103/PhysRevB.75.224305 PACS number(s): 43.20.+g, 43.58.+z, 68.60.Bs, 42.25.Hz

\section{INTRODUCTION}

The propagation of classical waves in a composite medium has been an interesting topic for the last decade mainly due to its technological applications. Since similarities and differences between the photonic and electronic band structures were discussed and summarized, ${ }^{1,2}$ photonic crystals have been intensively investigated both from the physical and application-oriented points of view. For these new materials, both theoretical and experimental results have shown the existence of ranges of frequencies where the propagation of electromagnetic waves is forbidden. The optical response of a photonic crystal with a triangular external shape (simulating an optical prism) has recently been studied both experimentally and theoretically. 3,4

In this time investigations have been extended to the propagation of sound waves in heterogeneous periodic structures made with materials of different elastic properties materials, the so-called sonic crystals (SCs). ${ }^{5}$ Several studies analyzed the applications of these materials with special acoustic properties. Some authors used the phenomenon of band gap in SCs to design acoustic filters. ${ }^{6,7}$ Recently, in order to increase the attenuation capability, some groups focused their work on a new class of materials based on SCs: the locally resonant sonic materials ${ }^{8}$ (LRSM) formed by soft and rigid elements. This combination is responsible for the appearance of attenuation peaks at very low frequencies, due to the resonant phenomena.

The interest in the similarities between optical and acoustical devices has increased in recent years since various authors ${ }^{9}$ demonstrated that it is possible to construct these SC-based devices for airborne sound with similar physical properties to analog optical systems. This behavior is possible due to the following properties: (i) sound transmission velocity inside the device is lower than that in air and (ii) the acoustic impedance of the device is similar to that of air. Certain authors ${ }^{10}$ have recently demonstrated that wave refraction is the main mechanism involved in this phenomenon in these systems. Several articles focused their investigations on the development of acoustic lenses based on SCs. Other authors ${ }^{11}$ introduced a new approach to designing highquality lenses based on SCs. Moreover, recent studies have demonstrated $^{12}$ that properly arranged arrays cannot only be- 
have as acoustic lenses but also the focusing effect can be well described by the lensmaker's formula.

One of the main parameters used to characterize SCs is the refraction index ${ }^{9} n$, especially if we want to use these materials to design acoustic refractive devices. These devices present a relevant property concerning their refraction index: they dramatically change the directions in which an incident wave travels in the second transmission band, leading to the apparent negative refraction of sound at the interface. ${ }^{13}$ This property has been previously reported for its optical analogs: the photonic crystals. ${ }^{14,15}$ Direct evidence of this effect has been shown by some authors ${ }^{16-19}$ in the range of ultrasounds. They use the negative refraction phenomena to focalize ultrasounds through a flat phononic crystal lens; i.e., they demonstrate the convergence of divergent sound beams without using curved-surface lenses. On the other hand, a number of research groups have demonstrated the possibility of focusing sound in the audible range, using acoustic lenses consisting of nonperiodic lattices of aluminum cylinders confined in a rectangular area without negative refraction..$^{11,20}$

If we consider the low-frequency range, the refraction index for airborne sound has usually been obtained, indirectly, by using the phase delay method. ${ }^{9,21,22}$ In this method, the phase information obtained is used to construct the acoustic dispersion relation wave vector $(\mathbf{k})$ versus frequency $(f)$ in the desired range of frequencies, obtaining the sound transmission velocity inside the SC. Subsequently, the refraction index is estimated as the ratio of sound velocities between outside (the air) and inside the SC. This technique is not appropriated for very low frequencies due to the erratic behavior of the phase in this range. Furthermore, the phase delay method considers the group velocity in the first transmission band due to its lineal behavior, in order to estimate the refraction index, obtaining a unique value for $\mathrm{n}$ in this range. It therefore seems of interest to study the behavior of the refraction index and to analyze its relationship with some typical SC parameters, with the aim of accurately characterizing these materials.

The goal of the present work is to study, numerically and experimentally, the behavior of the phase refractive index in the first transmission band, defined as the ratio of velocities, for bidimensional SCs, and to analyze their relationship with some typical SC parameters. For this purpose we have used an interferometric method based on the acoustical analog of an optical device called Fresnel's biprism ${ }^{23}$ (FB). Our experimental results are in good agreement with the numerical simulations that have been performed. Moreover, we have compared our results with those obtained by the phase delay method and we conclude that ours is more efficient, much simpler to perform, and more accurate than the phase delay method in estimating the acoustical refraction index. Finally, we show the nonlinear behavior of the refraction index at a very-low-frequency range (below $900 \mathrm{~Hz}$ ). This shows an unexpected behavior of the first transmission band, quite different from that obtained using other methods.

\section{THEORETICAL APPROACH}

In optics, the FB consists of two thin prisms joined at the bases and presents the property of producing an interference
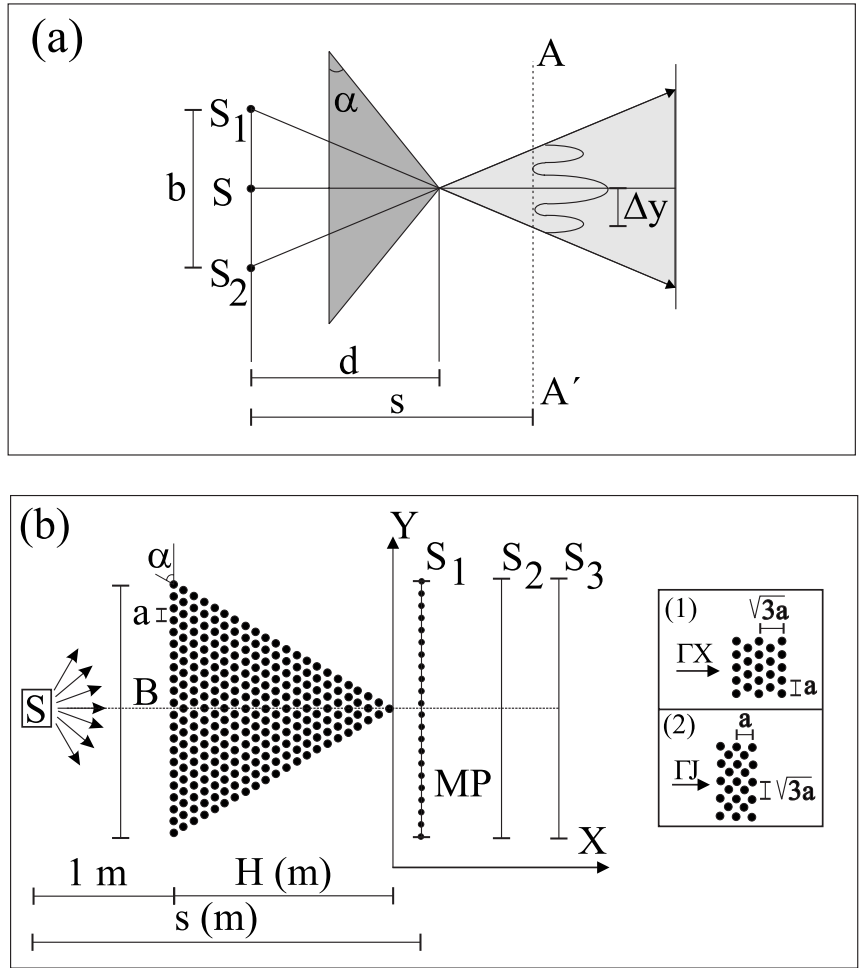

FIG. 1. (a) Optical Fresnel biprism. (b) Sonic Fresnel biprism constructed to obtain the refraction index in the $\Gamma X$ high-symmetry direction for a triangular array with lattice constant $a=6.35 \mathrm{~cm}$ together with the experimental setup used. In the inset, configurations used to construct sonic FB: (1) triangular array at $0^{\circ}$ ( $\Gamma X$ direction) and (2) triangular array at $30^{\circ}$ ( $\Gamma J$ direction). In both configurations, $a=6.35 \mathrm{~cm}$.

pattern: when a light wave impinges on the FB [Fig. 1(a)], a part of the incident wave is refracted downward and another part upward, creating an interference region. This behavior is equivalent to considering two coherent virtual sources $S_{1}$ and $S_{2}$, whose separation is $b$. That let the wave from the two images interfere. The separation of the virtual sources can be expressed in terms of the prism angle $\alpha$, as

$$
b=2 d \tan \left\{2 \arcsin \left[n \sin \left(\frac{\alpha}{2}\right)\right]-\alpha\right\},
$$

where $d$ is the distance from the source to the vertex of the FB and $n$ is the refraction index. On the other hand, the separation of fringes in the interference pattern created in a screen $A A^{\prime}$ is given by

$$
\Delta y=\frac{s}{b} \lambda=\frac{s c}{b f},
$$

where $c$ is the sound velocity in air and $f$ is the frequency. From Eqs. (1) and (2) it is possible to obtain the exact relation between the refraction index and the separation of fringes for a given wavelength:

$$
n=\frac{1}{\sin \left(\frac{\alpha}{2}\right)}\left(\sin \left\{\frac{1}{2}\left[\arctan \left(\frac{s c}{\Delta y 2 d f}\right)+\alpha\right]\right\}\right) \text {. }
$$


Taking into account that the designs of acoustical refractive devices have been based on SCs, we have constructed a bidimensional sonic FB with rigid cylinders in air. An example of the corresponding structure is shown in Fig. 1(b). Obviously, due to the different characteristics of the acoustic and electromagnetic fields, the analogy between both FB cannot be total. In order to calculate numerically the pressure field behind the sonic FB, we solve the wave equation using the finite-element method (FEM). This method was developed for the numerical discretization of continuous problems in bounded domains. ${ }^{24}$ From the mathematical point of view, it is a tool for solving problems with partial differential equations. In fact, these problems with complicated geometries are difficult to express by means of elementary functions. In this case, the methodology has two steps: (i) it is necessary to describe the geometry and generate a mesh for this in order to discretize the continuous problem and (ii) to build an equation for the discrete approximation of the solution. Finally some boundary conditions will be introduced which allow us to achieve a solution to the problem.

For our numerical simulation, we use a triangular mesh in the zone where we want to calculate the acoustic field. In the interference zone the mesh will be the densest region in order to obtain higher precision. We use approximately $10^{6}$ mesh elements to solve the wave equation in all the analyzed space.

In the case of rigid cylinders a sound hard boundary (wall) has been used; i.e., the velocity of the air particles is zero in the wall of the cylinder,

$$
\left.\vec{n} \cdot \vec{\nabla} p\right|_{\text {cylinder }}=0,
$$

and in the exterior walls of our system, we consider finiteimpedance conditions. So we can achieve the continuity conditions

$$
\left.\left[\vec{n} \cdot\left(\frac{1}{\rho_{0}} \vec{\nabla} p\right)-\frac{i \omega p}{Z}\right)\right|_{\text {wall }}=0,
$$

where $\rho_{0}$ and $Z$ are the density and the impedance of air, respectively.

The punctual noise source used in our experiments was implemented with the FEM using the next boundary condition

$$
\begin{aligned}
-\vec{n} & {\left[\left(\frac{1}{\rho_{0}}\right) \nabla p\right]+\left(i k+\frac{1}{2 r}\right) \frac{p}{p_{0}} } \\
& =\left[\left(i k+\frac{1}{2 r}\right) \frac{p}{p_{0}}-i(\vec{k} \cdot \vec{n})\right] p_{0} e^{-i(\vec{k} \cdot \vec{r}) / \rho_{0}} .
\end{aligned}
$$

The value of the pressure field in decibels is given by

$$
L_{\text {theo }}(x, y)=20 \log _{10}[|P(x, y)| / \delta\rfloor,
$$

where $\delta$ is an adjustable parameter that takes into account the fact that the incident pressure is not the unity in the experiments and $P(x, y)$ is obtained numerically by FEM. For each frequency we calculate the pressure map produced by the sonic FB and perform a transversal cut at a given distance from the acoustical source. The result is an interference
TABLE I. Characteristics of the sonic Fresnel biprism used.

\begin{tabular}{lcc}
\hline \hline & $\Gamma X$ direction & $\Gamma J$ direction \\
\hline No. of cylinders & 253 & 464 \\
$H(\mathrm{~m})$ & 1.155 & 0.9602 \\
$B(\mathrm{~m})$ & 1.334 & 3.135 \\
$\alpha(\mathrm{deg})$ & 60 & 30 \\
\hline \hline
\end{tabular}

sound pattern. From the transversal cuts we can estimate the separation of the fringes and finally, using Eq. (3), we can deduce numerically the refraction index for the FB.

\section{EXPERIMENTAL SETUP}

The experiments were performed in an echo-free chamber of dimensions $8 \times 6 \times 3 \mathrm{~m}^{3}$. We constructed two samples with triangular symmetry in order to analyze the refraction index along the two high-symmetry directions in the Brillouin zone $(\Gamma X$ and $\Gamma J)$ of the SC considered. The two samples are built up by hanging hollow aluminum cylinders in a frame with a triangular pattern with lattice constant $a$ $=6.35 \mathrm{~cm}$. The frame can rotate around the vertical axis, so the direction of the wave vector $(k)$ is perpendicular to the cylinder axis. Cylinders with diameter $D=4 \mathrm{~cm}$ and $1 \mathrm{~m}$ length were used to construct the refractive devices. The size of the sonic FB constructed is large enough to guarantee the study in the low-frequency region. In Table I we show the main characteristics of the sonic biprism constructed. For all the experiments a small speaker emitting continuous white noise was used so that the generated wave has a privileged direction of incidence, perpendicular to the base of the sonic FB. The signal transmitted throughout this refractive device was registered by a microphone and recorded in a dualchannel signal analyzer type B\&K 2148.

Figure 1(b) shows the experimental setup used. The source was placed at $1 \mathrm{~m}$ from the sonic FB. In order to obtain some pressure patterns of interference on a plane perpendicular to the cylinder axis, we fixed three different distances $s\left(s_{1}=2.455 \mathrm{~m}, s_{2}=3 \mathrm{~m}\right.$, and $\left.s_{3}=3.6 \mathrm{~m}\right)$. At each distance $s$, we measured perpendicularly to the acoustic field propagation ( $Y$ axis) at intervals of $10 \mathrm{~cm}$. In each transversal cut we considered 21 measurement points (MPs). As a result of the measurements we obtain at each point and for a given frequency the root-mean-squared (rms) pressure $P_{\text {rms }}(x, y)$. In order to express this in decibels we used a reference pressure $P_{\text {ref }}=20 \mu \mathrm{Pa}$. We thus define the experimental pressure in dBs by $P_{\text {expt }}=20 \log _{10}\left[P_{\text {rms }}(x, y) / P_{\text {ref }}\right]$.

\section{RESULTS AND DISCUSSION}

Due to the fact that the acoustical properties of SCs depend on several parameters, we analyzed their relationship with the refraction index $n$. The parameters considered were frequency, direction of the incident sound along the two high-symmetry directions in the Brillouin zone, and the filling factor (FF). Numerical calculations were achieved on a sonic FB made with two-dimensional (2D) SC arranged in a 
(a)
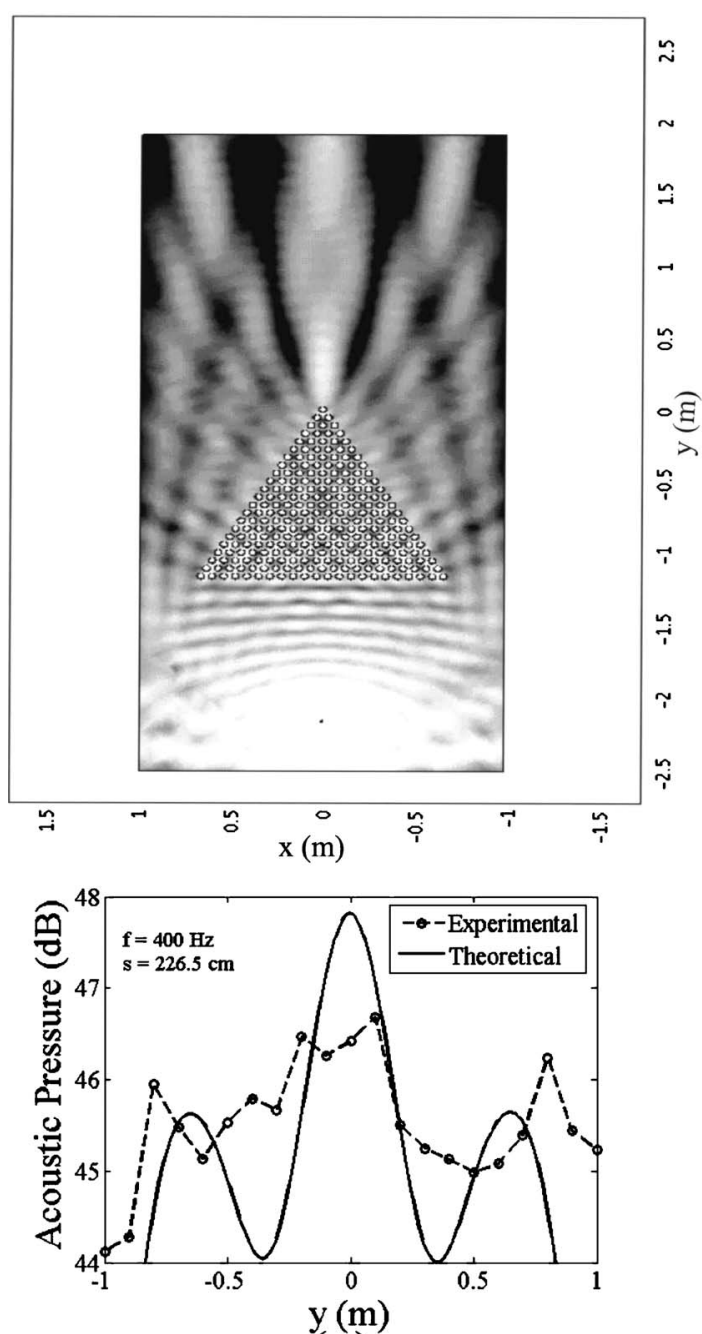

(c)

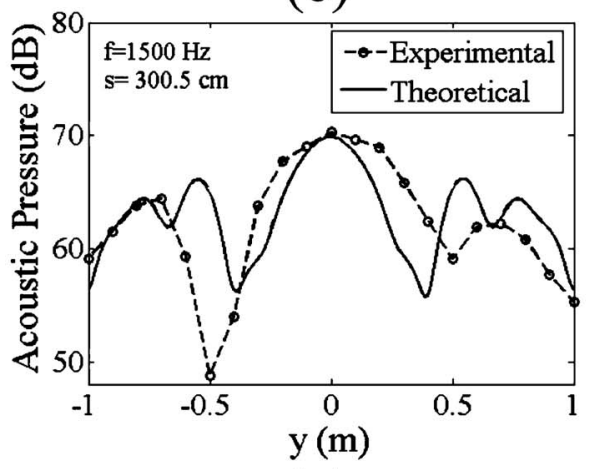

(e) (b)
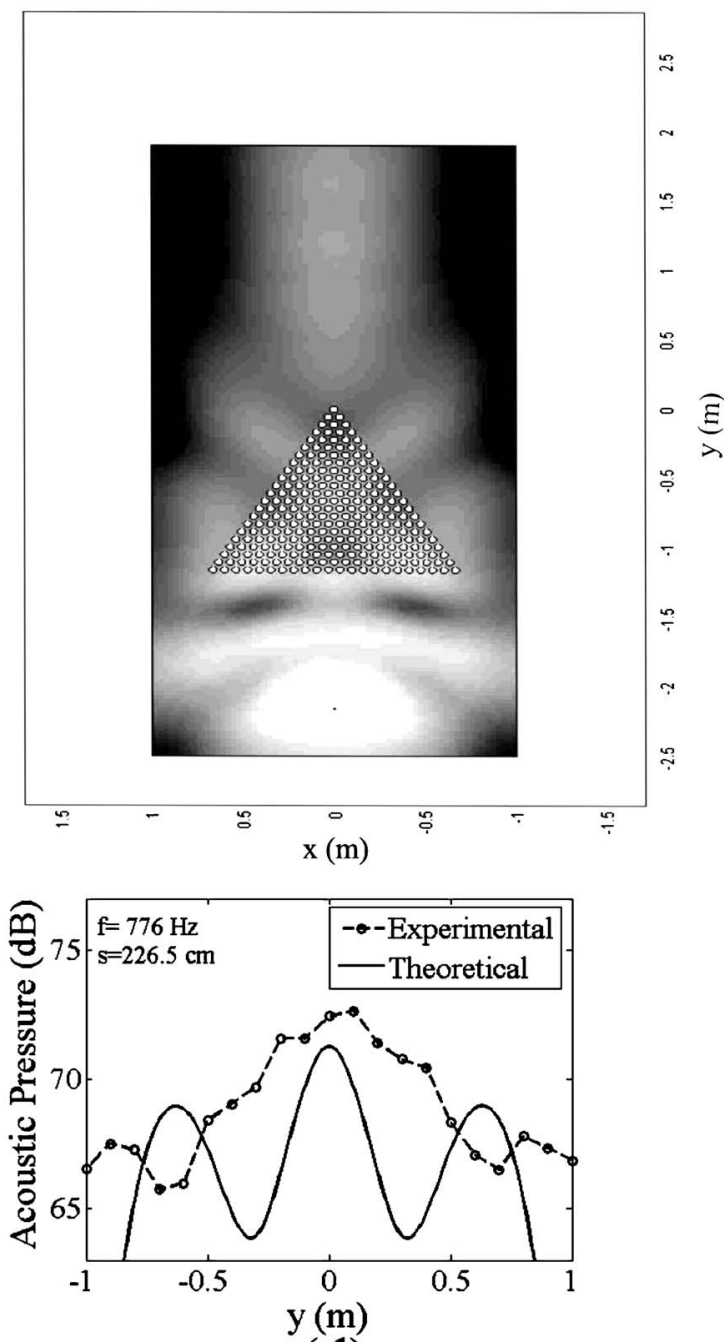

(d)

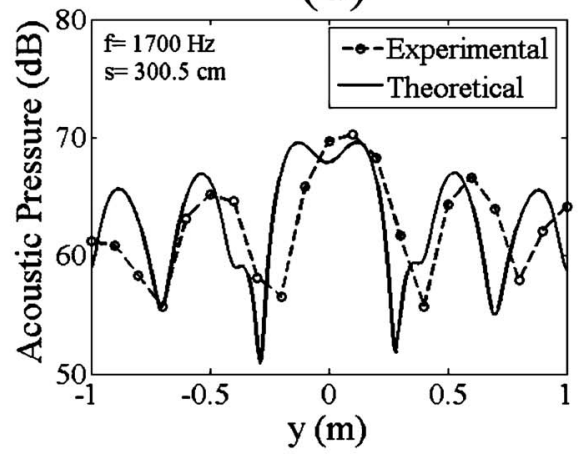

(f)

FIG. 2. Interferometric pressure pattern obtained with a sonic FB made with triangular array along the $\Gamma X$ direction. (a), (b) Calculated pressure map at $400 \mathrm{~Hz}$ and $1700 \mathrm{~Hz}$. (c), (d), (e), (f) Calculated and experimental transversal cuts of the pressure maps at different frequencies and distances.

triangular pattern with a lattice parameter of $6.35 \mathrm{~cm}$. Two different sonic FBs were used in order to analyze the refraction index along the two high-symmetry directions $\Gamma X$ and $\Gamma J$ [see Fig. 1(c)]. The diameter of the cylinders $(D)$ used throughout the numerical calculations was $4 \mathrm{~cm}$.
We focused our attention on the first transmission band, specifically in the range of frequencies between 330 and $2200 \mathrm{~Hz}$, since for frequencies lower than $300 \mathrm{~Hz}$ the size of the sonic FB is not large enough to obtain the searching pattern and the interference effect vanishes. For frequencies 


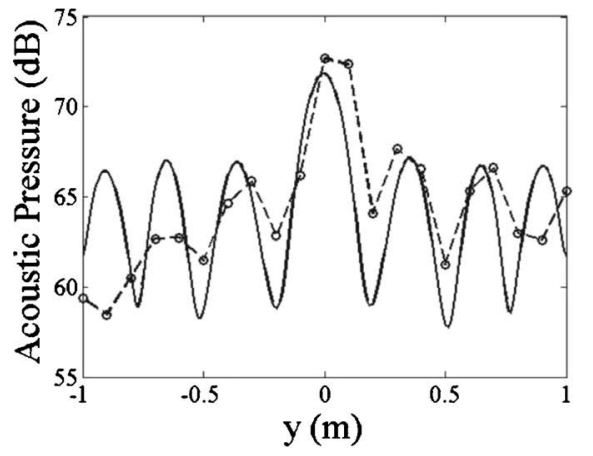

(a)

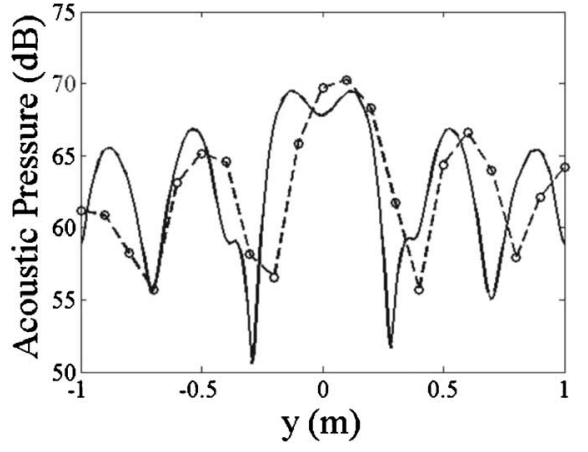

(b)

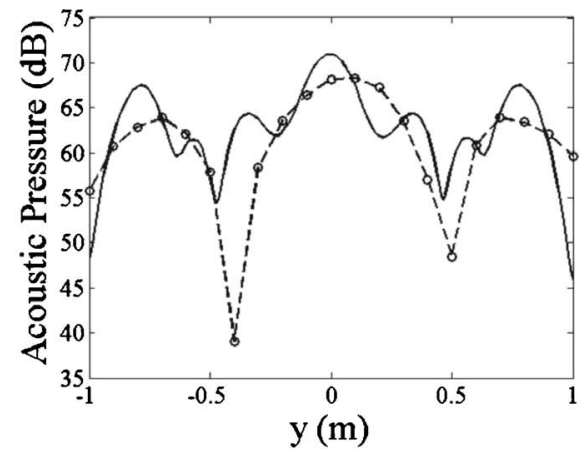

(c)

FIG. 3. Interference pressure patterns obtained for three different distances $s\left(s_{1}=2.455 \mathrm{~m}, s_{2}=3 \mathrm{~m}, s_{3}=3.6 \mathrm{~m}\right)$ at frequency $f$ $=1700 \mathrm{~Hz}$.

higher than $2200 \mathrm{~Hz}$ no interference effect appears, due to the proximity of the first gap at the two high-symmetry directions of the SC chosen.

The experimental results obtained with sonic FBs described above show the existence of an interference pattern in agreement with the numerical results predicted by FEM. Figure 2 shows an example of an interferometric pressure pattern obtained with a sonic FB made with a triangular array at $0^{\circ}$ ( $\Gamma X$ direction). In Figs. 2(a) and 2(b) we can see two examples of the pressure maps obtained for two frequencies $f_{1}=400 \mathrm{~Hz}$ and $f_{2}=1700 \mathrm{~Hz}$, with the interference pattern created behind the device. In Figs. 2(c) and 2(f) we show some transversal cuts of the pressure field in the interference region for several frequencies and distances $s$. In these cuts we can clearly see a pattern of interference fringes separated by a distance $\Delta y$. Furthermore, we can observe the inverse relation between the frequency and the separation of the interference fringes, $\Delta y$, in accordance with Eq. (2)-i.e., the higher the frequency, the lower the separation between fringes. The differences observed between numerical and experimental results in the Figs. 2(e) and 2(f) can be explained by the shift of the microphone out of the plane considered to measure the interference pattern, perpendicular to the direction of incidence. Moreover, in the case of Figs. 2(c) and 2(d) (very low frequencies), the sound interferes with both the periodicity of the array and the size of the sonic FB employed. The distortion due to the size of the sonic FB produces a deformation of the acoustic wave, and the interference pattern is clearly affected. Even so, the positions of the experimental and theoretical fringes in the interference pattern are in good agreement and, most of all, for each low frequency considered, the distances between fringes, theoretical and experimentally, are similar. This fact is essential to calculate the refraction index as we explain below and to guarantee the goodness of our theoretical model.

The interference patterns obtained allow us to estimate the value of the refraction index as a function of the frequency, measuring the distance between two consecutive fringes $(\Delta y)$ and applying expression (3), in the same way as with the optical FB. Note that the distance obtained between two consecutive maximum values of acoustic pressure in the pattern of interferences $(\Delta y)$ is directly related to the distance $s$, so that we can measure this difference for any $s$ and we can get the same refraction index for any couple of $\Delta y$ and $s$. Thus, the same pattern of interference for a given crystal gives a unique value of $n$. In Fig. 3 we show the interference pressure patterns obtained in our work for a frequency $f$ $=1700 \mathrm{~Hz}$ and for the three different distances $s$ considered. We obtain the same value of $n$ for the three different cuts showed (see Table II). As one can see in the figure, the comparison between theory and experiment is better for the case of the smallest $s$. This fact means that, from the experimental point of view, small $s$ means small $\Delta y$, and the mesh used for the measurements must be small enough to see clearly the interference pattern, as we have done in our experiments.

Specifically, we estimate the refraction index using our method in opposition to the phase delay method used in Refs. 9 and 12. In both cases, using refractive devices based on different SCs, the authors estimate the refraction index as a single value for the first transmission band using the phase velocity obtained from the phase delay. They obtain the index refraction values for the frequencies $f_{1}=1500 \mathrm{~Hz}$ and $f_{2}=1700 \mathrm{~Hz}$, respectively. Note that these frequencies are located in the range of frequencies where the behavior of $n$ is linear. Using the sonic FB method we obtain the same values for the refraction index as those estimated in Refs. 9 and 12. Figures 2(e) and 2(f) show the interference pressure pattern obtained with the sonic FB method for the frequencies under study. These results confirm the validity of our method of

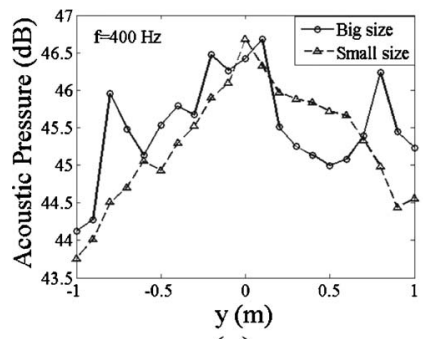

(a)

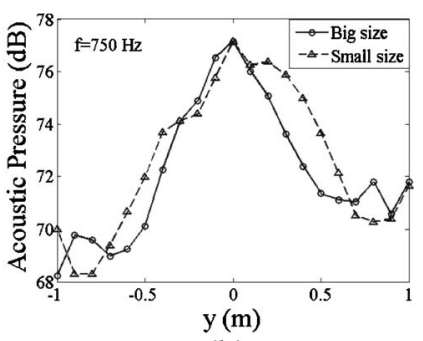

(b)
FIG. 4. Experimental interference patterns obtained with both sizes of sonic $\mathrm{FBs}$, for the $\Gamma X$ direction and for two frequencies $f_{1}=400 \mathrm{~Hz}$ and $f_{2}=750 \mathrm{~Hz}$. (a) $f_{1}=400 \mathrm{~Hz}$ and $\Gamma X$ direction and (b) $f_{2}=750 \mathrm{~Hz}$ and $\Gamma X$ direction. 
TABLE II. Theoretical and experimental values of $n$ obtained for a frequency $f=1700 \mathrm{~Hz}$ and for the three cuts shown in Fig. 1.

\begin{tabular}{ccc}
\hline \hline$s(\mathrm{~m})$ & $n$ (theoretical) & $n$ (experimental) \\
\hline 2.455 & 1.27 & $1.3 \pm 0.1$ \\
3.0 & 1.27 & $1.3 \pm 0.1$ \\
3.6 & 1.24 & $1.3 \pm 0.1$ \\
\hline \hline
\end{tabular}

estimating the refraction index for different SCs independently of their external shape. Moreover, the sonic FB method allows us to obtain the refraction index as a function of the frequency and, consequently, in the very-lowfrequency range.

In order to analyze the influence of the size of the sonic FB in the estimated interference patterns, and consequently in the values of the refraction index, we have constructed two news sonic FBs. The new devices are equal to those used up to now, but with a smaller size. The main characteristics of these sonic FBs are for the $\Gamma J$ direction, No. of cylinders $=251, B=127 \mathrm{~cm}, H=62 \mathrm{~cm}$, and for the $\Gamma X$ direction, No. of cylinders $=78, B=74 \mathrm{~cm}, H=65 \mathrm{~cm}$. In Fig. 4 we show the interference patterns obtained experimentally with both sizes of sonic FBs for two low frequencies and for the $\Gamma X$ direction. One can see that the distances between the fringes are similar in both cases. The observed difference is the increase of the clarity of the interference pattern in the large sonic FBs, but in any case the size of the FB used has had an influence in the distances between fringes and, consequently, in the value of the refraction index calculated. This fact and the good agreement between the experimental and theoretical data shown in Fig. 5 means that the size of the devices used do not have a real influence in the value of the refraction index obtained.

Figure 5 shows the estimated value of the refraction index $n$ as a function of the frequency for the triangular array considered and for their two high-symmetry directions in the Brillouin zone. Moreover, we have included the band structure of the SC used in an inset to clarify the range of frequencies included in the first band of transmission. We show a comparison between measurements (symbols) and simulations by FEM (lines). Excellent agreement between the theory and experiments can be observed. It is interesting to note this coincidence in the low-frequency range, where an increase in the value of the refraction index was predicted by the calculations. In the literature, we have not found any study on the refraction index in this range and, consequently, we cannot compare our results. We want to point out that the dependence of $n$ with frequency is not linear and, therefore, the first band of transmission obtained is not linear either, in opposition to the results predicted by the theoretical model of the band structures. 5 This disagreement between results could be due to the assumptions used in the theoretical model, which were not used in the studies made with real sonic crystals. We therefore consider our method to be more accurate.

On the other hand, Fig. 5 shows the existence of different curves for each high-symmetry direction. This result gives the behavior of the sonic crystal as a function of the frequency: at the range of frequencies between 300 and $1000 \mathrm{~Hz}$ (low frequencies) one can see a small anisotropy of the crystal-i.e., the existence of different values of $n$ depending on the incident direction of the wave. However, at the range of frequencies between 1100 and $2000 \mathrm{~Hz}$ we can see the quasilinear behavior of $n$. This means that sonic crystals are isotropic in this range. Consequently, we can define an effective refraction index in this range and compare it with the phase delay method, obtaining the same results, as described above.

Finally, with regard to the behavior of the refraction index with the (FF), Fig. 6 gives the calculated values obtained by FEM for the triangular array used in this work and in the $\Gamma X$ direction, varying the filling fraction from 0.04 to 0.8 . We show this behavior for three different frequencies in the range under study: $700 \mathrm{~Hz}$ (low frequency), $1250 \mathrm{~Hz}$ (middle frequency), and $2000 \mathrm{~Hz}$ (high frequency).

It can be seen in Fig. 6 that for FF values lower than 0.5 the behavior of the refraction index $n$ is explained by the relationship $1 / f$; i.e., a high value of $n$ is expected in the low-frequency range and a low value of $n$ is expected in the high-frequency region, according to Eq. (3). Nevertheless,

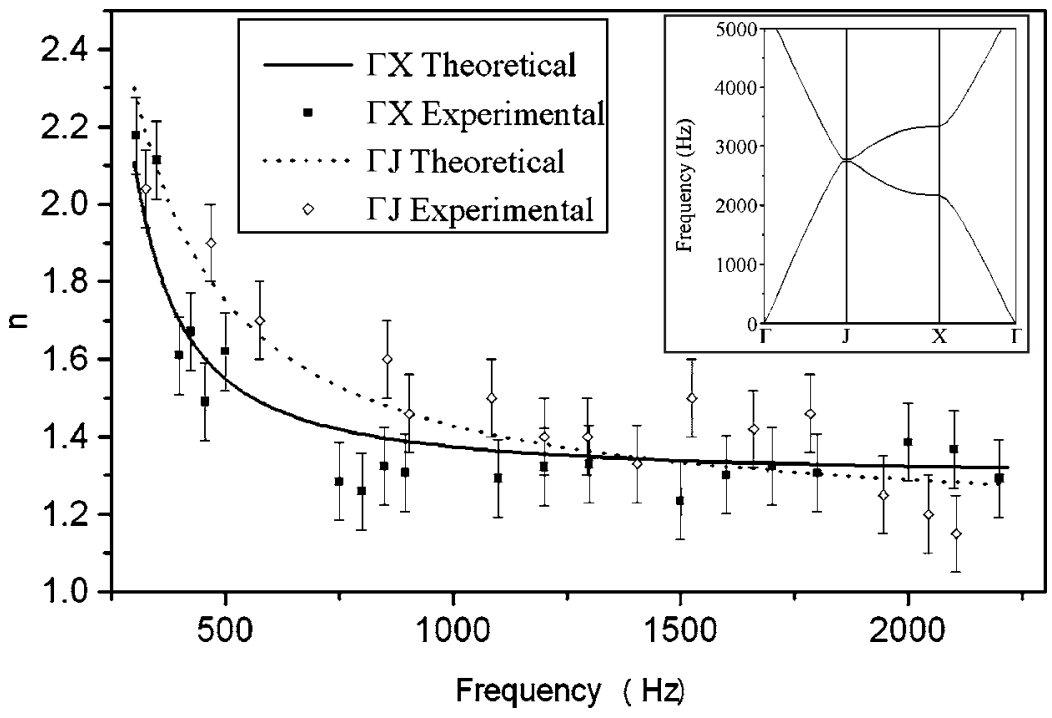

FIG. 5. Dispersion relation obtained with the sonic FB method for the triangular array and for the two high-symmetry directions in the Brillouin zone. The symbols mark the experimental values obtained, and the dotted and solid lines define those estimated with simulations by FEM. The inset shows the band structure of the SC used (triangular array and lattice constant $a=6.35 \mathrm{~cm}$ ). 


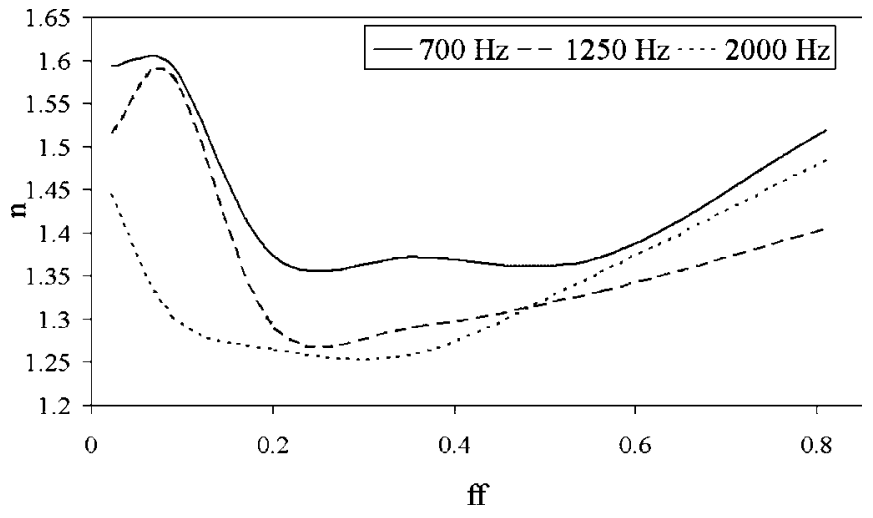

FIG. 6. Filling factor dependence of the refraction index for a triangular array with lattice constant $a=6.35 \mathrm{~cm}$, along the $\Gamma X$ direction, calculated by FEM for three frequencies $700 \mathrm{~Hz}$ (solid line), $1250 \mathrm{~Hz}$ (dashed line), and $2000 \mathrm{~Hz}$ (dotted line).

Fig. 6 also shows a different shape of the curve of the high frequency considered $(2000 \mathrm{~Hz})$ for FF values higher than 0.5 . An explanation of this behavior could be given by the fact that the width of the gap grows with the value of the FF, ${ }^{5}$ and this high frequency could be near the first gap, causing a distortion of the interference effect and, consequently, of the value of $n$.

\section{SUMMARY}

In this paper, we present a direct method of obtaining the phase refraction index within the first transmission band in materials called sonic crystals, as opposed to the other indirect method (phase delay method). We consider our method to be more efficient, much simpler to perform, and more accurate. Furthermore, we analyze the relationship between the refraction index and different SC parameters, such as type of array, filling fraction, and direction of the incident sound along the two high-symmetry directions.

Our method was developed by an analogy with the Fresnel biprism used in optics and was therefore called the sonic Fresnel biprism method. We provide experimental and theoretical results, using a numerical method based on FEM. Obviously, the analogy between the two devices cannot be total due to the differences between the two fields and due to the fact that in optics the devices are formed by an infinite number of scatterers and sonic crystals have a finite number. But the behavior of both devices presents a certain amount of agreement: first, the equations developed for the optical case can be used in the acoustic case, and second, using the interference pattern obtained, it is possible to establish directly the phase refraction index in both cases. In addition, in the acoustic case there is considerable agreement between the theoretical and experimental results and with those obtained by other authors.

We obtain interesting conclusions about the phase refraction index using our method: (i) the nonlinear behavior of the dispersion relation $n$ (refraction index) versus frequency below $900 \mathrm{~Hz}$. This shows the behavior of the first transmission band to be different from that obtained using the band theory and therefore the possible nonvalidity of the hypothesis used in the low-frequency range in this theory when we are analyzing real sonic crystals. (ii) The results obtained show a small amount of anisotropy in the low-frequency range, but the behavior of the crystal is isotropic at other frequency ranges within the first band, which is just the range in which we can compare our results with those obtained by other authors.

Even so, more studies must be done in order to develop a model to explain the results obtained in this work.

\section{ACKNOWLEDGMENTS}

This work has been partially supported by the Spanish Ministry of Science and Education (Project No. MAT200703097) and partially supported by Generalitat Valenciana (Project GV/2007/191). We would like to thank the $\mathrm{R}+\mathrm{D}$ $+\mathrm{i}$ Linguistic Assistance Office at the Universidad Politécnica de Valencia for their help in revising this paper.
${ }^{1}$ E. Yablonovitch, Phys. Rev. Lett. 58, 2059 (1987).

${ }^{2}$ S. John, Phys. Rev. Lett. 58, 2486 (1987).

${ }^{3}$ S. Y. Lin, V. M. Hietala, L. Wang, and E. D. Jones, Opt. Lett. 21, 1771 (1996).

${ }^{4}$ J. Bravo-Abad, T. Ochiai, and J. Sánchez-Dehesa, Phys. Rev. B 67, 115116 (2003).

${ }^{5}$ J. V. Sánchez-Pérez, D. Caballero, R. Martínez-Sala, C. Rubio, J. Sánchez-Dehesa, F. Meseguer, J. Llinares, and F. Gálvez, Phys. Rev. Lett. 80, 5325 (1998).

${ }^{6}$ M. S. Kushwaha, Appl. Phys. Lett. 70, 3218 (1997).

${ }^{7}$ J. V. Sánchez-Pérez, C. Rubio, R. Martínez-Sala, R. SánchezGrandía, and V. Gómez, Appl. Phys. Lett. 81, 5240 (2002).

${ }^{8}$ Z. Liu, X. Zhang, Y. Mao, Y. Y. Zhu, Z. Yang, C. T. Chan, and P. Sheng, Science 289, 1734 (2000).

${ }^{9}$ F. Cervera, L. Sanchis, J. V. Sánchez-Pérez, R. Martínez-Sala, C.
Rubio, F. Meseguer, C. López, D. Caballero, and J. SánchezDehesa, Phys. Rev. Lett. 88, 023902 (2002).

${ }^{10}$ A. Hakansson, J. Sánchez-Dehesa, F. Cervera, F. Meseguer, L. Sanchis, and J. Llinares, Phys. Rev. E 71, 018601 (2005).

${ }^{11}$ A. Hakansson, J. Sánchez-Dehesa, and L. Sanchis, Phys. Rev. B 70, 214302 (2004).

${ }^{12}$ C. Kuo and Z. Ye, J. Phys. D 37, 2155 (2004).

${ }^{13}$ J. H. Page, A. Sukhovich, S. Yang, M. L. Cowan, F. Van Der Biest, A. Tourin, M. Fink, Z. Liu, C. T. Chan, and P. Sheng, Phys. Status Solidi A 241, 3454 (2004).

${ }^{14}$ M. Notomi, Phys. Rev. B 62, 10696 (2002).

${ }^{15}$ E. Cubukun, K. Aydin, E. Ozbay, S. Foteinopoulus, and C. M. Sokoulis, Nature (London) 423, 604 (2003).

${ }^{16}$ X. Zhang and Z. Liu, Appl. Phys. Lett. 85, 341 (2004).

${ }^{17}$ C. Qiu, X. Zhang, and Z. Liu, Phys. Rev. B 71, 054302 (2005). 
${ }^{18}$ L. Feng, X. P. Liu, Y. B. Chen, Z. P. Huang, Y. W. Mao, Y. F. Chen, J. Zi, and Y. Y. Zhu, Phys. Rev. B 72, 033108 (2005).

${ }^{19}$ M. Ke, Z. Liu, C. Qiu, W. Wang, J. Shi, W. Wen, and P. Sheng, Phys. Rev. B 72, 064306 (2005).

${ }^{20}$ A. Hakansson, F. Cervera, and J. Sánchez-Dehesa, Appl. Phys. Lett. 86, 054102 (2005).

${ }^{21}$ C. Rubio, D. Caballero, J. V. Sánchez-Pérez, R. Martínez-Sala, J.
Sánchez-Dehesa, F. Meseguer, and F. Cervera, J. Lightwave Technol. 17, 2202 (1999).

${ }^{22}$ W. M. Robertson and J. F. Rudy III, J. Acoust. Soc. Am. 104, 694 (1998).

${ }^{23}$ E. Hecht, Óptica, 3rd ed. (Addison-Wesley, Madrid, 2000).

${ }^{24}$ F. Ihlenburg, Finite Elements Analysis of Acoustic Scattering (Springer-Verlag, Berlin, 1998). 